PROCEEDINGS OF THE

AMERICAN MATHEMATICAL SOCIETY

Volume 135, Number 10, October 2007, Pages 3309-3316

S $0002-9939(07) 08868-5$

Article electronically published on June 21, 2007

\title{
THE WEIL-PETERSSON GEOMETRY ON THE THICK PART OF THE MODULI SPACE OF RIEMANN SURFACES
}

\author{
ZHENG HUANG
}

(Communicated by Richard A. Wentworth)

\begin{abstract}
In the thick part of the moduli space of Riemann surfaces, we show that the sectional curvature of the Weil-Petersson metric is bounded independently of the genus of the surface.
\end{abstract}

\section{INTRODUCTION}

Results on the asymptotic geometry of the Weil-Petersson metric on Teichmüller space are presented in this paper. However, there is no curve pinching in the setting of this paper. We restrict ourselves to the thick part of the moduli space, where there is a positive lower bound on the least hyperbolic length of any short closed curves on the surface. We obtain boundedness of the Riemannian curvatures on the thick part of the moduli space with respect to the genus $g$, by considering surfaces with large genera.

The motivation of this paper is the recent consideration of articles [12] and [13] of Maryam Mirzakhani, where she studied the hyperbolic lengths of simple closed geodesics on a Riemann surface and calculated the Weil-Petersson volume of the moduli space of Riemann surfaces. The Weil-Petersson volume grows exponentially in $g$ (see also [23]), in particular, a rational multiple of $\pi^{6 g-6+2 n}$, where $n$ is the number of punctures on the surface. Thus it is natural to ask how the curvatures vary in terms of $g$. We find, in Theorem 1.2, that the absolute value of the WeilPetersson sectional curvature is bounded with respect to $g$, in the thick part of the moduli space.

Let $\Sigma$ be a smooth, oriented, closed Riemann surface of genus $g$; we always assume $g>1$. The study of conformal structures, or equivalently hyperbolic metrics, on a compact Riemann surface naturally leads to the classical Teichmüller theory.

Teichmüller space $\mathcal{T}_{g}$ is the space of conformal structures on $\Sigma$, modulo an equivalent relationship, where two conformal structures $\sigma$ and $\rho$ are equivalent if there is a biholomorphic map between $(\Sigma, \sigma)$ and $(\Sigma, \rho)$, in the homotopy class of the identity. Riemann's moduli space $\mathcal{M}_{g}$ of Riemann surfaces is obtained as the quotient of Teichmüller space by the mapping class group.

Teichmüller space $\mathcal{T}_{g}$ is known ([1]) to be a complex manifold of complex dimension $3 g-3$, and the cotangent space at $\Sigma$ is identified with $Q D(\Sigma)$, the space

Received by the editors March 16, 2006 and, in revised form, July 26, 2006.

2000 Mathematics Subject Classification. Primary 30F60, 32G15.

(C)2007 American Mathematical Society

Reverts to public domain 28 years from publication 
of holomorphic quadratic differentials. One also identifies the tangent space with $H B(\Sigma)$, the space of harmonic Beltrami differentials.

We recall that the Weil-Petersson cometric on Teichmüller space is defined on $Q D(\Sigma)$ by the $L^{2}$-norm:

$$
\|\phi\|_{W P}^{2}=\int_{\Sigma} \frac{|\phi|^{2}}{\sigma} d z d \bar{z}
$$

where $\sigma|d z|^{2}$ is the hyperbolic metric on $\Sigma$. By duality, we obtain a Riemannian metric on the tangent space of $\mathcal{T}_{g}$ : for $\mu \in H B(\Sigma)$, we have

$$
\|\mu\|_{W P}^{2}=\int_{\Sigma}|\mu|^{2} \sigma d z d \bar{z}=\int_{\Sigma}|\mu|^{2} d A .
$$

The differential geometry of this metric on Teichmüller space has been extensively studied. Ahlfors showed that the Ricci curvature, the holomorphic sectional curvature and scalar curvatures are negative ([1, 2]). Royden showed that the holomorphic sectional curvature is bounded away from zero and conjectured an explicit bound $\frac{-1}{2 \pi(g-1)}([15])$, later proved by Wolpert $([24)$.

Throughout this paper, we denote $l_{0}(\sigma)$ as the systole, the length of the shortest closed geodesic, of the surface $\Sigma$. As an important invariant of hyperbolic metrics, the systole is related to several important aspects of the Weil-Petersson geometry, such as the injectivity radius and curvatures near the compactification divisor, as seen in [25], [26], 7], 8].

In this paper, we assume there is a positive lower bound $r_{0}$ on the injectivity radius, denoted by $i n j_{\sigma}(\Sigma)$, of $\Sigma$; therefore the systole $l_{0}(\sigma)$ also has a positive lower bound. We call the thick part of the moduli space as the compact subset of the moduli space where $i n j_{\sigma}(\Sigma)>r_{0}>0$, i.e., the thick part of the moduli space consists of fat surfaces.

Our first result concerns the Weil-Petersson holomorphic sectional curvature on a large part of the moduli space:

Theorem 1.1. There exists a positive constant $C_{1}$, independent of $g$, such that the Weil-Petersson holomorphic sectional curvature $K_{h}$ satisfies

$$
-C_{1}<K_{h}<\frac{-1}{2 \pi(g-1)}
$$

on the thick part of the moduli space.

Tromba and Wolpert proved that the Weil-Petersson sectional curvatures are negative ([19], 24]). For the asymptotics of the sectional curvatures, much consideration has been on the Weil-Petersson geometry near the compactification divisor. It is shown that there are directions (near the compactification divisor) in which the sectional curvatures have no negative upper bound ([7]), nor lower bound ([] [18), for fixed $g$.

When the surface is away from the compactification divisor, from a compactness argument, all curvatures are bounded and the bounds are in terms of $g$ and $l_{0}(\sigma)$. In this paper, we find that the curvatures are bounded, independent of the genus $g$, i.e.,

Theorem 1.2. On the thick part of the moduli space, there is a positive constant $\mathrm{C}_{2}$, independent of the genus $\mathrm{g}$, such that the sectional curvature $K$ of the WeilPetersson metric satisfies

$$
-C_{2}<K<0 .
$$


As an easy application, we also find the following estimates of the Ricci curvature and the scalar curvature:

Theorem 1.3. On the thick part of the moduli space, there are positive constants $C_{3}, C_{4}$, independent of the genus $g$, such that the Ricci curvature of the WeilPetersson metric satisfies

$$
-C_{3} g<\operatorname{Ric}\left(d_{W P}\right)<\frac{-1}{2 \pi(g-1)} .
$$

and the scalar curvature $s$ satisfies

$$
-C_{4} g^{2}<s<-\frac{9 g-6}{4 \pi} .
$$

The main technique involved is the analysis of harmonic maps between hyperbolic surfaces. The influence of Eells and Sampson's pioneering work ([5]) is reflected in many perspectives of Teichmüller theory; for example, see [9], 4], 20], 21. 11, and many others. One often finds that many functions associated to harmonic maps satisfy some geometric differential equations, where one can apply techniques in geometric analysis.

Here is the outline of the paper. In $\S 2$, we give a quick exposition of the background and introduce our notions. We prove our main theorems in $\S 3$.

The author is grateful to Maryam Mirzakhani for bringing this problem to his attention, and to Xiaodong Wang and Mike Wolf for their very insightful comments and generous help. He also thanks the referee(s) for careful reading and valuable suggestions.

\section{BACKGROUND}

In this paper, let $\Sigma$ be a fixed, compact, smooth, oriented surface of genus $g>1$. We also denote $\sigma|d z|^{2}$ as a hyperbolic metric on $\Sigma$, for conformal coordinates $z$. On $\left(\Sigma, \sigma|d z|^{2}\right)$, we denote the Laplacian as

$$
\Delta=\frac{4}{\sigma} \frac{\partial^{2}}{\partial z \partial \bar{z}},
$$

with nonpositive eigenvalues.

Teichmüller space is a complex manifold with the mapping class group acting by biholomorphisms, of complex dimension $3 g-3$, the number of independent closed curves in any pair-of-pants decomposition of the surface.

The Weil-Petersson metric is invariant under the action of the mapping class group; hence it descends to a metric on the moduli space. It is shown that the Weil-Petersson metric is Kählerian ([1]), with negative sectional curvature ([19, 24]). The Weil-Petersson Riemannian curvature tensor is given as the TrombaWolpert formula ([19], 24]):

$$
R_{\alpha \bar{\beta} \gamma \bar{\delta}}=\int_{\Sigma} D\left(\mu_{\alpha} \bar{\mu}_{\beta}\right) \mu_{\gamma} \bar{\mu}_{\delta} d A+\int_{\Sigma} D\left(\mu_{\alpha} \bar{\mu}_{\delta}\right) \mu_{\gamma} \bar{\mu}_{\beta} d A
$$

where $\Delta$ is the Laplacian for the hyperbolic metric $\sigma$ on the surface, and $D=$ $-2(\Delta-2)^{-1}$ is a self-adjoint, compact operator. Those $\mu$ 's in the formula are tangent vectors, i.e., harmonic Beltrami differentials. There is a natural pairing between $Q D(\Sigma)=\left\{\phi(z) d z^{2}\right\}$ and $H B(\Sigma)=\left\{\mu(z) \frac{d \bar{z}}{d z}\right\}$ :

$$
\left\langle\phi d z^{2}, \mu \frac{d \bar{z}}{d z}\right\rangle=\operatorname{Re} \int_{\Sigma} \phi \mu d z d \bar{z} .
$$

A remarkable property of the Weil-Petersson metric is that it is incomplete ([10], 22]). This is caused by pinching off at least one short closed geodesic on the surface. The Weil-Petersson completion modulo the mapping class group is topologically the Deligne-Mumford compactification of the moduli space. The compactification 
divisor thus consists of a union of lower-dimensional Teichmüller spaces, where each such space consists of noded Riemann surfaces, obtained by pinching nontrivial short closed geodesics on the surface $([3,10])$. Therefore the compactification divisor can be described via the systole $l_{0}(\sigma)$ as the set $\left\{l_{0}(\sigma)=0\right\}$.

Since we will analyze harmonic maps between compact hyperbolic surfaces, we recall some fundamental facts here. For a Lipschitz map $w:\left(\Sigma, \sigma|d z|^{2}\right) \rightarrow$ $\left(\Sigma, \rho|d w|^{2}\right)$, where $\sigma|d z|^{2}$ and $\rho|d w|^{2}$ are hyperbolic metrics on $\Sigma$, and $z$ and $w$ are conformal coordinates on $\Sigma$, one follows Sampson ([16]) to define

$$
\mathcal{H}(z)=\frac{\rho(w(z))}{\sigma(z)}\left|w_{z}\right|^{2}, \quad \mathcal{L}(z)=\frac{\rho(w(z))}{\sigma(z)}\left|w_{\bar{z}}\right|^{2} .
$$

We call $\mathcal{H}(z)$ the holomorphic energy density, and $\mathcal{L}(z)$ the anti-holomorphic energy density. Then the energy density function of $w$ is simply $e(w)=\mathcal{H}+\mathcal{L}$, and the total energy is then given by

$$
E(w, \sigma, \rho)=\int_{\Sigma} e \sigma|d z|^{2} .
$$

We also note that the Jacobian determinant relative to the $\sigma$ metric is therefore given by $J(z)=\mathcal{H}(z)-\mathcal{L}(z)$.

The map $w$ is called harmonic if it is a critical point of this energy functional, i.e., if it satisfies the Euler-Lagrange equation:

$$
w_{z \bar{z}}+\frac{\rho_{w}}{\rho} w_{z} w_{z \bar{z}}=0 .
$$

The $(2,0)$ part of the pullback $w^{*} \rho$ is the so-called Hopf differential:

$$
\phi(z) d z^{2}=\left(w^{*} \rho\right)^{(2,0)}=\rho(w(z)) w_{z} \bar{w}_{z} d z^{2} .
$$

It is routine to check that $w$ is harmonic if and only if $\phi d z^{2} \in Q D(\Sigma)$, and $w$ is conformal if and only if $\phi=0$.

In our situation, there is a unique harmonic map $w:(\Sigma, \sigma) \rightarrow(\Sigma, \rho)$ in the homotopy class of the identity; moreover, this map $w$ is a diffeomorphism with positive Jacobian $J$, and $\mathcal{H}>0$ ([5, 6], [16, [17]).

A key observation to link the harmonic maps to Teichmüller theory is that one obtains a map from Teichmüller space to $Q D(\Sigma)$, for some fixed hyperbolic metric $\sigma$. More specifically, this map sends any hyperbolic metric on $\Sigma$ to a holomorphic quadratic differential associated to the unique harmonic map in the homotopy class of the identity. This map is a diffeomorphism ([16, 20]).

\section{ProOF OF MAIN THEOREMS}

Let $\mu=\mu(z) \frac{d \bar{z}}{d z}$ be a unit Weil-Petersson normed harmonic Beltrami differential; thus $\int_{\Sigma}|\mu|^{2} d A=1$. The Weil-Petersson holomorphic sectional curvature in the direction of $\mu$ is given by

$$
K_{h}=-2 \int_{\Sigma} D\left(|\mu|^{2}\right)|\mu|^{2} d A .
$$

Its upper bound, in terms of the genus, is known, proved by Wolpert:

Lemma $3.1([25]) . K_{h}<-\frac{1}{2 \pi(g-1)}$.

Remark 3.2. This upper bound holds without restriction on the systole. Since all sectional curvatures are negative, so the Ricci curvature and scalar curvature are bounded from above by $-\frac{1}{2 \pi(g-1)}$, and $-\frac{3(3 g-2)}{4 \pi}$, respectively $([25])$.

We now show the following pointwise estimate on $|\mu(z)|$, where the tangent vector $\mu(z) \frac{d \bar{z}}{d z}$ is normalized to have unit Weil-Petersson norm, and we shall apply this estimate to prove our main theorems. 
Theorem 3.3. For $\mu(z) \frac{d \bar{z}}{d z} \in H B(\Sigma)$ with $\|\mu\|_{W P}=1$, there exists a positive constant $h_{0}$, independent of $g$, such that $|\mu(z)| \leq h_{0}$, for all $z \in \Sigma$, where the surface $\Sigma$ is in the thick part of the moduli space.

Proof. Recall that $\mu(z) \frac{d \bar{z}}{d z}$ is a harmonic Beltrami differential, hence is a symmetric tensor given as $\bar{\phi}\left(d s^{2}\right)^{-1}$ for $\phi$ a holomorphic quadratic differential with at most simple poles at the cusps and $d s^{2}$ the hyperbolic metric tensor ([23]). Since the surface $\Sigma$ lies in the thick part of the moduli space, hence there are no cusps and this holomorphic quadratic differential $\phi=\phi(z) d z^{2}$ has no poles.

Note that $\phi \in Q D(\Sigma)$, as stated in the previous section. Then by a theorem of Wolf $([20])$, there exists a hyperbolic metric $\rho$ on the surface $\Sigma$, and a unique harmonic map $w:(\Sigma, \sigma) \rightarrow(\Sigma, \rho)$, such that $\phi$ is the Hopf differential associated to this harmonic map $w$, i.e., $\phi(z) d z^{2}=\rho(w(z)) w_{z} \bar{w}_{z} d z^{2}$.

Much of our study will be analyzing this harmonic map $w$. Note that even though $\operatorname{inj}_{\sigma}(\Sigma)>r_{0}>0$, the metric $\rho$ might not lie in the same thick part of the moduli space. We recall that the holomorphic and anti-holomorphic energy density functions of $w$ are defined as $\mathcal{H}(z)=\frac{\rho(w(z))}{\sigma(z)}\left|w_{z}\right|^{2}$, and $\mathcal{L}(z)=\frac{\rho(w(z))}{\sigma(z)}\left|w_{\bar{z}}\right|^{2}$, respectively. Since the map $w$ is a diffeomorphism with positive Jacobian determinant, we have $\mathcal{H}(z)>\mathcal{L}(z) \geq 0$.

We also find that $\mathcal{H} \mathcal{L}=\frac{|\phi|^{2}}{\sigma^{2}}=|\mu|^{2}$, so the zeros of $\mathcal{L}$ are the zeros of $|\mu|$, or equivalently, the zeros of $\phi$.

Let $\nu$ be the Beltrami differential of the map $w$, defined by $\nu=\frac{w_{z} d \bar{z}}{w_{z} d z}$. It measures the failure of $w$ to be conformal, and since $J>0$, we have $|\nu|<1$.

One easily finds that $|\nu|^{2}=\frac{\mathcal{L}}{\mathcal{H}}$; therefore,

$$
|\mu|=\sqrt{\mathcal{H} \mathcal{L}}=\mathcal{H}|\nu|<\mathcal{H}
$$

Thus it suffices to estimate $\mathcal{H}(z)$ to bound $|\mu|$ pointwisely.

Let $z_{0} \in \Sigma$ such that $\mathcal{H}\left(z_{0}\right)=\max _{z \in \Sigma} \mathcal{H}(z)$. We follow a calculation of Schoen and Yau to define a local one-form $\theta=\sqrt{\sigma(z)} d z$, and find ([17]):

$$
\left|w_{\theta}\right|^{2}=\frac{\rho}{\sigma}\left|w_{z}\right|^{2}=\mathcal{H}(z)
$$

and

$$
\Delta\left|w_{\theta}\right|^{2}=4\left|w_{\theta \theta}\right|^{2}+2 J\left|w_{\theta}\right|^{2}-2\left|w_{\theta}\right|^{2} .
$$

We rewrite this as

$$
\Delta \mathcal{H}=4\left|w_{\theta \theta}\right|^{2}+2 J \mathcal{H}-2 \mathcal{H}>-2 \mathcal{H} .
$$

Therefore $\mathcal{H}$ is a subsolution to an elliptic equation $(\Delta+2) f=0$.

Recalling that $\operatorname{inj}_{\sigma}(\Sigma)>r_{0}>0$, we embed a hyperbolic ball $B_{z_{0}}\left(\frac{r_{0}}{2}\right)$ into $\Sigma$, centered at $z_{0}$ with radius $\frac{r_{0}}{2}$. Morrey's theorem ([14, theorem 5.3.1) on subsolutions of elliptic differential equations guarantees that there is a constant $C\left(r_{0}\right)$ such that

$$
\mathcal{H}\left(z_{0}\right)=\sup _{B_{z_{0}}\left(\frac{r_{0}}{4}\right)} \mathcal{H}(z) \leq C\left(r_{0}\right) \int_{B_{z_{0}}\left(\frac{r_{0}}{2}\right)} \mathcal{H}(z) \sigma d z d \bar{z}
$$

Another consequence of formula (3) is the Bochner identity (see [17]), as now $\log \mathcal{H}$ is well defined:

$$
\Delta \log \mathcal{H}=2 \mathcal{H}-2 \mathcal{L}-2
$$


The minimal principle implies that $\mathcal{H}(z) \geq 1$ for all $z \in \Sigma$, and we find

$$
\int_{\Sigma} \mathcal{L} d A \leq \int_{\Sigma} \mathcal{H} \mathcal{L} d A=\int_{\Sigma}|\mu|^{2} d A=\|\mu\|_{W P}=1 .
$$

It is not hard to see that we can actually bound the total energy of this harmonic map $w$ from above, in terms of the genus. More precisely, we recall that $w$ is a diffeomorphism, so

$$
\int_{\Sigma}(\mathcal{H}-\mathcal{L}) d A=\int_{\Sigma} J d A=\operatorname{Area}(w(\Sigma))=4 \pi(g-1) ;
$$

therefore the total energy satisfies

$$
\begin{aligned}
E(w) & =\int_{\Sigma} e(w) d A=\int_{\Sigma}(\mathcal{H}+\mathcal{L}) d A \\
& =\int_{\Sigma}(\mathcal{H}-\mathcal{L}) d A+2 \int_{\Sigma} \mathcal{L} d A \\
& \leq \operatorname{Area}(\Sigma, \sigma)+2 \\
& =4 \pi(g-1)+2 .
\end{aligned}
$$

Therefore

$$
\begin{aligned}
\int_{B_{z_{0}}\left(\frac{r_{0}}{2}\right)} \mathcal{H}(z) \sigma d z d \bar{z} & <\int_{B_{z_{0}}\left(\frac{r_{0}}{2}\right)}(\mathcal{H}(z)+\mathcal{L}(z)) \sigma d z d \bar{z} \\
& =E(w)-\int_{\Sigma \backslash B_{z_{0}}\left(\frac{r_{0}}{2}\right)} e(z) \sigma d z d \bar{z} \\
& \leq 4 \pi(g-1)+2-\left(4 \pi(g-1)-A_{1}\left(B_{z_{0}}\left(\frac{r_{0}}{2}\right)\right)\right) \\
& =A_{1}\left(B_{z_{0}}\left(\frac{r_{0}}{2}\right)\right)+2,
\end{aligned}
$$

where $A_{1}\left(B_{z_{0}}\left(\frac{r_{0}}{2}\right)\right)$ is the hyperbolic area of the ball $B_{z_{0}}\left(\frac{r_{0}}{2}\right)$.

We set $h_{0}=C\left(r_{0}\right)\left(A_{1}\left(B_{z_{0}}\left(\frac{r_{0}}{2}\right)\right)+2\right)$. Then $h_{0}$ is independent of the genus $g$, as it is obtained from a local estimate in a geodesic ball. Therefore,

$$
|\mu(z)|<\mathcal{H}(z) \leq \mathcal{H}\left(z_{0}\right)<h_{0},
$$

for all $z \in \Sigma$.

Remark 3.4. The assumption of the surface lying in the thick part of the moduli space is essential to this argument, since we used an estimate in an embedded geodesic ball.

As an application of this estimate, we can prove Theorem 1.1 easily.

Proof of Theorem 1.1. Recall that the operator $D=-2(\Delta-2)^{-1}$ is self-adjoint and $\|\mu\|_{W P}=1$ :

$$
\left|K_{h}\right|=2 \int_{\Sigma} D\left(|\mu|^{2}\right)|\mu|^{2} d A<2 h_{0}^{2} \int_{\Sigma} D\left(|\mu|^{2}\right) d A=2 h_{0}^{2} .
$$

We now shift our attention to general Weil-Petersson sectional curvatures and to the proof of Theorem 1.2.

Proof of Theorem 1.2. We recall from the previous section that the Riemannian curvature tensor of the Weil-Petersson metric is given by ([19], 24]):

$$
R_{\alpha \bar{\beta} \gamma \bar{\delta}}=\int_{\Sigma} D\left(\mu_{\alpha} \bar{\mu}_{\beta}\right) \mu_{\gamma} \bar{\mu}_{\delta} d A+\int_{\Sigma} D\left(\mu_{\alpha} \bar{\mu}_{\delta}\right) \mu_{\gamma} \bar{\mu}_{\beta} d A,
$$


where the $\mu$ 's in the formula are harmonic Beltrami differentials, and $D$ again is the operator $-2(\Delta-2)^{-1}$.

To calculate the sectional curvature, we choose two arbitrary orthonormal harmonic Beltrami differentials $\mu_{0}$ and $\mu_{1}$. In other words, we have

$$
\int_{\Sigma}\left|\mu_{0}\right|^{2} d A=\int_{\Sigma}\left|\mu_{1}\right|^{2} d A=1 \text { and } \int_{\Sigma} \mu_{0} \bar{\mu}_{1} d A=0 .
$$

Then the Gaussian curvature of the plane spanned by $\mu_{0}$ and $\mu_{1}$ is (24])

$$
\begin{aligned}
K\left(\mu_{0}, \mu_{1}\right) & =\frac{1}{4}\left(R_{0 \overline{1} 0 \overline{1}}-R_{0 \overline{1} 1 \overline{0}}-R_{1 \overline{0} 0 \overline{1}}+R_{1 \overline{0} 1 \overline{0}}\right) \\
& =\operatorname{Re}\left(\int_{\Sigma} D\left(\mu_{0} \bar{\mu}_{1}\right) \mu_{0} \bar{\mu}_{1} d A\right)-\frac{1}{2} \operatorname{Re}\left(\int_{\Sigma} D\left(\mu_{0} \bar{\mu}_{1}\right) \mu_{1} \bar{\mu}_{0} d A\right) \\
& -\frac{1}{2} \int_{\Sigma} D\left(\left|\mu_{1}\right|^{2}\right)\left|\mu_{0}\right|^{2} d A .
\end{aligned}
$$

From ([24, lemma 4.3) and Hölder's inequality, we have

$$
\begin{aligned}
\left|\operatorname{Re}\left(\int_{\Sigma} D\left(\mu_{0} \bar{\mu}_{1}\right) \mu_{0} \bar{\mu}_{1} d A\right)\right| & \leq \int_{\Sigma}\left|D\left(\mu_{0} \bar{\mu}_{1}\right)\right|\left|\mu_{0} \bar{\mu}_{1}\right| d A \\
& \leq \int_{\Sigma} \sqrt{D\left(\left|\mu_{0}\right|^{2}\right)} \sqrt{D\left(\left|\mu_{0}\right|^{2}\right)}\left|\mu_{0} \bar{\mu}_{1}\right| d A \\
& \leq \int_{\Sigma} D\left(\left|\mu_{0}\right|^{2}\right)\left|\mu_{1}\right|^{2} d A=\int_{\Sigma} D\left(\left|\mu_{1}\right|^{2}\right)\left|\mu_{0}\right|^{2} d A
\end{aligned}
$$

and similarly

$$
\left|\int_{\Sigma} D\left(\mu_{0} \bar{\mu}_{1}\right) \mu_{1} \bar{\mu}_{0} d A\right| \leq \int_{\Sigma} D\left(\left|\mu_{1}\right|^{2}\right)\left|\mu_{0}\right|^{2} d A
$$

Therefore

$$
\left|K\left(\mu_{0}, \mu_{1}\right)\right| \leq 2 \int_{\Sigma} D\left(\left|\mu_{1}\right|^{2}\right)\left|\mu_{0}\right|^{2} d A .
$$

We apply Theorem 3.3 to find that there is an $h_{0}>0$, independent of $g$, such that $\left|\mu_{0}\right|<h_{0}$. So $|K|<2 h_{0}^{2} \int_{\Sigma} D\left(\left|\mu_{1}\right|^{2}\right) d A=2 h_{0}^{2}$.

It is now straightforward to see that Theorem 1.3 holds since all sectional curvatures are negative.

\section{REFERENCES}

[1] L. Ahlfors, Some remarks on Teichmüller's space of Riemann surfaces, Ann. of Math. (2)74 (1961) 171-191 MR0204641 (34:4480)

[2] L. Ahlfors, Curvature properties of Teichmüller space, J. Anal. Math. 9 (1961) 161-176 MR0136730 (25:192)

[3] L. Bers, Spaces of degenerating Riemann surfaces, Discontinous groups and Riemann surfaces, Ann. of Math. Studies, 79, Princeton University Press, Princeton, New Jersey, 1974 MR0361051 (50:13497)

[4] C. Earle, J. Eells, A fibre bundle description of Teichmüller theory, J. Diff. Geom. 3 (1969) 19-43 MR.0276999(43:2737a)

[5] J. Eells, J. Sampson, Harmonic mappings of Riemannian manifolds, Amer. J. Math. 86 (1964) 109-160 MR0164306 (29:1603)

[6] P. Hartman, On homotopic harmonic maps, Can. J. Math. 19 (1967) 673-687 MR0214004 $(35: 4856)$

[7] Z. Huang, Asymptotic flatness of the Weil-Petersson metric on Teichmüller space, Geom. Dedi., 110 (2005) No. 1, 81-102 MR2136021

[8] Z. Huang, On asymptotic Weil-Petersson geometry of Teichmüller space of Riemann surfaces, Asian J. Math., to appear 
[9] J. Jost, Two dimensional geometric variational problems, Wiley-Interscience, 1990 MR:1100926 (92h:58045)

[10] H. Masur, The extension of the Weil-Petersson metric to the boundary of Teichmüller space, Duke Math. J., 43 (1976) 623-635 MR0417456 (54:5506)

[11] Y. Minsky, Harmonic maps, length, and energy in Teichmüller space, J. Diff. Geom., 35 (1992) 151-217 MR.1152229 (93e:58041)

[12] M. Mirzakhani, Simple geodesics and Weil-Petersson volumes of moduli spaces of bordered Riemann surfaces, Ann. of Math.(2), to appear

[13] M. Mirzakhani, Weil-Petersson volumes and intersection theory on the moduli space of curves, Preprint, 2004

[14] C. Morrey, Multiple integrals in the calculus of variations, Springer-Verlag, 1966 MR0202511 $(34: 2380)$

[15] H. Royden, Intrinsic metrics on Teichmüller space, Proc. Int. Cong. Math. 2 (1974) 217-221 MR0447636 (56:5946)

[16] J. Sampson, Some properties and applications of harmonic mappings, Ann. Sci. Ecole Norm. Sup. 4 (1978) 211-228 MR510549 (80b:58031)

[17] R. Schoen, S. Yau, On univalent harmonic maps between surfaces, Invent. Math. 44 (1978) 265-278 MR0478219 (57:17706)

[18] S. Trapani, On the determinant of the bundle of meromorphic quadratic differentials on the Deligne-Mumford compactification of the moduli space of Riemann surfaces, Math. Ann. 293 (1992) 681-705 MR:1176026 (93g:58157)

[19] A. Tromba, On a natural algebraic affine connection on the space of almost complex structures and the curvature of Teichmüller space with respect to the Weil-Petersson metric, Manuscr. Math. 56 (1986) 475-497 MR860734 (88c:32034)

[20] M. Wolf, The Teichmüller theory of harmonic maps, J. Diff. Geom. 29 (1989) 449-479 MR.982185 (90h:58023)

[21] M. Wolf, Infinite energy harmonic maps and degeneration on hyperbolic surfaces in moduli space J. Diff. Geom., 33 (1991) 487-539 MR.1094467 (92b:58055)

[22] S. Wolpert, Noncompleteness of the Weil-Petersson metric for Teichmüller space, Pac. J. Math. 61 (1975) 573-577 MR0422692 (54:10678)

[23] S. Wolpert, On the homology of the moduli space of stable curves, Ann. of Math.(2) 118 (1983) 491-523 MR727702 (86h:32036)

[24] S. Wolpert, Chern forms and the Riemann tensor for the moduli space of curves, Invent. Math. 85 (1986) 119-145 MR842050 (87j:32070)

[25] S. Wolpert, Geometry of the Weil-Petersson completion of Teichmüller space, Surveys in Differential Geometry, VIII: Papers in Honor of Calabi, Lawson, Siu and Uhlenbeck, Inter. Press 2003 MR2039996 (2005h:32032)

[26] S. Wolpert, Weil-Petersson perspectives, preprint, 2005

Department of Mathematics, University of Michigan, Ann Arbor, Michigan 48109

E-mail address: zhengh@umich.edu 